\title{
A THREE AND FIVE COLOR THEOREM
}

\author{
FRANK R. BERNHART ${ }^{1}$
}

\begin{abstract}
Let $f$ be a face of a plane graph $G$. The Three and Five Color Theorem proved here states that the vertices of $G$ can be colored with five colors, and using at most three colors on the boundary of $f$. With this result the well-known Five Color Theorem for planar graphs can be strengthened, and a relative coloring conjecture of Kainen can be settled except for a single case which happens to be a paraphrase of the Four Color Conjecture. Some conjectures are presented which are intermediate in strength to the Four Color Conjecture and the Three and Five Color Theorem.
\end{abstract}

In this paper we prove a coloring theorem for planar graphs. Two important corollaries are derived: a strengthened form of the Five Color Theorem, and a relative coloring conjecture of Kainen [1].

To color a graph means to assign colors to the vertices in such a way that each pair of adjacent vertices receive distinct colors. A coloring is a $n$-coloring if at most $n$ different colors are used. A plane graph is a graph drawn in the plane subject to the usual restrictions; each connected region of the complement is a face. The set of vertices and edges incident with a face is the boundary of the face. A subgraph of a graph is induced (or "full") if it contains an edge whenever it contains the endpoints of the edge.

The main result and the two corollaries are as follows:

I. If $G$ is a plane graph with a face f, then $G$ has a 5-coloring which is also a 3-coloring of the boundary of $f$.

II. Let $G$ be a graph which becomes planar if one edge is removed; then $G$ can be 5-colored.

III. If $H$ is a connected induced subgraph of planar graph $G$, then any 2-coloring of $H$ can be extended to a 5-coloring of $G$.

Before presenting the proofs, a few observations and lemmas are stated. It is clearly no loss of generality if all graphs considered are strict, that is, have no loops or multiple edges. If $H$ is a subgraph of $G$, let $N(H)$ be the set of vertices of $G$ that are not in $H$ but are adjacent to some vertex of $H$. If $f$ is a face of $G$, write $V(f, H)$ for the set of vertices common to $H$ and the boundary of $f$, and write $\bar{V}(f, H)$ for the set of vertices of $H$ that lie on the

Received by the editors December 25, 1973 and, in revised form, August 12, 1974. $05 \mathrm{C} 10$.

AMS (MOS) subject classifications (1970). Primary 05C15, 55A15; Secondary

${ }^{1}$ This research was done while the author was a postdoctoral fellow in Combinatorics and Optimization at the University of Waterloo, Waterloo, Ontario, Canada. 
boundary of the unique face $f^{\prime}$ of graph $H$ containing $f$. Clearly $\bar{V}(f, H) \supseteq V(f, H)$ and $V(f, G)$ is the set of boundary vertices of $f$. Let the vertices of $G$ be $V(G)$.

If the graph $G$ can be drawn so that for some face $f$ we have $V(G)=$ $V(f, G)$, then $G$ is outer planar. If $H$ is a connected subgraph and $G-H$ is the graph that remains when the vertices of $H$ and all incident edges are removed, then there is a face $f$ of $G-H$ such that $N(H) \subseteq V(f, G-H)$. It follows that the abstract graph $G$ can be drawn as an outer planar graph just in case $G \circ w$ is planar, where $G \circ w$ is formed from $G$ by adding a new vertex $w$ and edges joining $w$ to every other vertex.

Since the complete graph on five vertices is not planar, whenever $v$ is a vertex of plane $G$ of degree $\operatorname{deg}(\nu) \geq 4$, some two of the vertices adjacent to $v$ cannot be adjacent to each other. And since we can insert a vertex $v$ into any face / and make $N(v)=V(f, G)$, the same is true for the vertices of any set $V(f, G)$ containing more than three members.

Write $C_{f}$ for the boundary of a face $f$ if it is a circuit. We can assume that $G$ is drawn with face $f$ on the outside of $C_{f}$. Call $G$ a near-triangulation with $C_{f}$ as boundary if each face inside $C_{f}$ (different than $f$ ) is bounded by a triangle. We will need the next three lemmas.

Lemma 1. Let $G$ be a strict plane graph with face f. There exists a strict plane near-triangulation $G^{\prime}$ with face $f^{\prime}$ such that $G$ is obtained from $G^{\prime}$ by deleting edges, and $V(f, g)=V\left(f^{\prime}, G^{\prime}\right)$.

Lemma 2. If $G$ is outer planar, then $G$ is 3-colorable.

Lemma 3. If $G$ is a strict near-triangulation with boundary $C_{f}$, then either $C_{f}$ contains a vertex $v$ with $\operatorname{deg}(v) \leq 4$, or there is a vertex $v$ inside $C_{f}$ with $\operatorname{deg}(v) \leq 5$.

We omit the details of the proof of Lemma 1. If some face $g \neq f$ is not a triangle, we can add an edge between two nonadjacent vertices. If the boundary of $f$ is not a circuit, we can add an edge in $f$ which joins vertices in different components of $G$, or two nonadjacent vertices that are common neighbors of a vertex $w$ and which lie in different components of $G-w$ ( $w$ is a cutpoint).

Lemma 2 follows from Lemma 1 by observing that Lemma 1 implies outer planar $G$ is a subgraph of a graph which is a triangulation (on one side) of a polygon, and that the latter are proved 3-colorable by an easy induction.

If Lemma 3 were not true, we would have a strict near-triangulation $G$ such that the degree of each $v$ is at least five, and at least six if $v$ is not on $C_{f}$. Add a vertex $v_{f}$ in face $f$ and join to the members of $V(f, G)$; then $v$ is the only vertex which can have degree less than six. This contradicts well-known deductions from Euler's formula for plane graphs. 
The main theorem is now proved by induction on the number of vertices. If plane $G$ has three vertices or less, then $G$ can be 3-colored. For $n \geq 4$ we assume that I holds for $G$ with less than $n$ vertices, and consider $G$ with $n$ vertices. We let $1,2,3,4,5$ be a standard set of five colors. Using Lemma 1 it is no loss of generality to assume that the boundary of $f$ is a circuit $C_{f}$, and that $G$ is a near-triangulation with boundary $C_{f}$.

${ }^{\circ} 1$. Suppose first that there is an edge $[u v]$ inside $C_{f}$ connecting vertices $u$ and $v$ on $C_{f}$. Then there are two paths $P_{1}$ and $P_{2}$ contained in $C_{f}$ with endpoints $u, v$. Let $C_{i}$ be the circuit $P_{i}+[u v]$, and let $H_{i}$ be the graph consisting of $C_{i}$ and all parts of $G$ lying inside $C_{i}$, for $i=1,2$. By induction and permutation of colors we can find colorings of the near-triangulations $H_{i}$ in $1,2,3,4,5$ which color the vertices in the sets $\bar{V}\left(f, H_{i}\right)$ in the colors $1,2,3$, and such that $u, v$ have the same color in each coloring. The two colorings may then be combined to give the required coloring for $G$.

In cases ${ }^{\circ} 2$ and ${ }^{\circ} 3$ it is assumed that $C_{f}$ has no diagonals.

${ }^{\circ} 2$. Let $v$ be a vertex interior to $C_{f}$, and having degree less than six. Thus $\operatorname{deg}(v)=3,4$, or 5 . If $\operatorname{deg}(v)=3$, form $H$ by deleting $v$, otherwise form $H$ by contracting to one vertex $v$ and two of its neighbors $u_{1}$ to $u_{2}$ which are nonadjacent. This generates, by induction, a coloring for $G$ with $v$ excepted in which the neighbors of $v$ are colored with at most four colors. This leaves a color for $v$.

3. Suppose that $C_{f}$ contains a vertex $v$ of degree $\operatorname{deg}(v) \leq 4$; form $H$ by removing $C_{f}$ and incident edges. Note that $N\left(C_{f}\right)$ contains at most two vertices of $H$ neighbor to $v$, and $N\left(C_{f}\right)=V\left(f^{\prime}, H\right)$ for some face $f^{\prime}$ of $H$. By induction and permutation of colors there is a coloring of $H$ with $1,2,3,4$, 5 so that $N\left(C_{f}\right)$ is colored with 3,4,5 and the vertex $v$ is not neighbor to a vertex colored 3. Let $v$ be colored 3, and then finish coloring $C_{f}$ using 1,2 . This gives the required coloring of $G$.

Theorem I is now proved. With almost no extra effort, we can prove that a stronger statement holds in a special case.

$I^{*}$. If $G$ is a plane graph with a face $f$, and the boundary vertices $V(f, G)$ induce a bipartite subgraph $H$, then $G$ has a 5-coloring which is also a 2 coloring of the boundary of $f$.

It is enough to observe $H$ lies in a face of $G-H$, and we can add a 5coloring of $G-H$ with colors 3, 4, 5 on $N(H)$, which exists by I, to a 2 coloring of $H$.

Now Theorem II is derived. Let $G$ be a plane graph, and $u, v$ be any two distinct vertices of $G$. Theorem I implies that there is a 5-coloring of $G-u$ such that the neighbors of $u$ are colored with three colors. Then $u$ can be given a color different from $v$ and from any of its neighbors, and that results in a 5-coloring of $G+[u v]$ with new edge $[u v]$. 
Define the skeuness $\mathrm{sk}(G)$ of a graph $G$ to be the least number of edges that need to be removed from $G$ in order to make the result planar. Then our Corollary II is equivalent to the statement that any graph $G$ with sk $(G) \leq$ 1 is 5-colorable. Recently Kainen has proved [2] $G$ is 5-colorable even when $\mathrm{sk}(G) \leq 2$. This is best possible since complete graph $K(6)$ has skewness three. The proof given above for Theorem I was in part suggested by Kainen's proof.

Next it is shown that I and III are easy corollaries of each other. Let $H$ be a connected induced subgraph of planar graph $G$, and suppose $H$ has been 2 -colored. If $H=G$, the coloring trivially extends to $G$. If $H \neq G$ then Theorem I is applicable to $G-H$, choosing for $f$ the face which includes $N(H)$ in its boundary. After permutation, we have a coloring of $G$ in which the colors of $H$ are 1,2 and the colors of $N(H)$ are 3, 4, 5. Hence III follows from I.

On the other hand, let $f$ be a face of plane $G$. Draw in the face $f$ a long enough circuit $C$ of even length so that each vertex in $V(f, G)$ can be joined to two consecutive vertices of $C$. In the resulting graph $G^{*}$ color $C$ with 1,2 and extend the coloring (using III) to $G^{*}$. This clearly gives a coloring of $G$ satisfying the requirement of $I$.

Theorem III is the case $k=2$ for a family $\{S(k): k=1,2,3, \ldots\}$ of relative coloring conjectures. Member $S(k)$ states that any coloring in $k$ colors of a connected induced subgraph $H$ of plane graph $G$ can be extended to a $(k+3)$-coloring of $G$. Relative coloring was introduced by Kainen [1]. The statement that $S(k)$ holds for all $k$ is the same as the Connected Relative Three Color Conjecture (CR3CC), which says that any given coloring of a connected induced subgraph of a plane graph will extend using at most three new colors. Kainen gave a simple contraction argument to show that (CR3CC) was equivalent to the Four Color Conjecture (4CC). It is easy to see that Theorem I implies $S(k)$ for $k \geq 2$, and that $S(1)$ is a trivial rewording of the (4CC), because $H$ must then consist of a single vertex. This tends to trivialize the equivalence between (CR3CC) and (4CC).

The situation was more interesting at the time Kainen's article was published. As is mentioned therein, I had an easy proof for $S(k)$ if $k \geq 3$, and it was hoped that this proof could be modified to take care of $S(2)$. That hope never materialized. The easy proof was as follows.

We assume without loss that $G$ and $H$ both are connected, and that $H \neq G$. Let $H$ be colored with $k \geq 3$ colors, take $1,2,3$ as three new colors, and let $4,5,6$ be any three of the first $k$ colors. Let $m=1,2$, $3, \cdots$ and define $U_{m}$ to be the set of vertices at distance $m$ from $H$. Let $H_{m}$ be the graph induced by $V(H)$ and all $U_{i}$ with $i<m$. Clearly $H_{m}$ is connected, $H_{1}=H$, and $N\left(H_{m}\right)=U_{m}$. If $U_{m}$ is not empty, then the graph induced by 
$U_{m}$ is outer planar, as $H_{m}$ is connected (see above). Color each nonempty $U_{m}$ with the three colors $1,2,3$ if $m$ is even, and with $4,5,6$ if $m$ is odd. This clearly gives the required coloring.

The equivalence of Theorem III, or $S(2)$, with Theorem I suggests a new family of conjectures.

Conjecture $(n)$. Let $G$ be a plane graph including faces $f_{i}(i=1,2$, $\cdots, n)$. Then there exists a 5-coloring of $G$ such that every face $f_{i}$ has a boundary colored with three colors.

Note that different $f_{i}$ need not use the same three colors. Generalizing on $n$ we have

(3\&5CC) Three and Five Color Conjecture. Every plane graph $G$ can be colored with five colors in such a way that the boundary of each face is 3-colored.

The (4CC) is equivalent to the following conjecture, which implies (3\&5CC):

(3\&4CC) Three and Four Color Conjecture. Each plane graph $G$ can be colored with four colors so that the boundary of each face is 3-colored.

The equivalence is obvious, since it is possible to insert a vertex in each face with more than three vertices on the boundary, and join it to all these vertices.

It may happen that $(3 \& 5 \mathrm{CC})$ is not hard to prove. However, the proof given for Theorem $\mathrm{I}$, which is the same as case $n=1$, does not seem to apply easily to case $n=2$. We notice however,

\section{Case $n=2$ of ( $3 \& 5 C C)$ implies the theorem of Kainen that} states that each graph of skewness at most 2 can be 5-colored.

Proof. We let $G$ be a planar graph, and $u_{1}, v_{1}, u_{2}, v_{2}$ be any four vertices. We want to show that $G+\left[u_{1} v_{1}\right]+\left[u_{2} v_{2}\right]$ can be 5-colored. If either $G+\left[u_{1} v_{1}\right]$ or $G+\left[u_{2} v_{2}\right]$ is planar, then the result follows from Theorem II. It is clearly no loss to assume $G$ is a triangulation of the plane. Suppose that $u_{1} u_{2} v_{1} v_{2}\left(u_{1}\right)$ is a circuit in $G$. The circuit must be a separating circuit. Form graphs $G_{1}$ and $G_{2}$ by replacing first one side, then the other of the circuit with edge $\left[u_{1} v_{1}\right]$. Theorem II applies to $G_{1}+$ $\left[u_{2} v_{2}\right]$ and $G_{2}+\left[u_{2} v_{2}\right]$, but since in both cases $u_{1}, u_{2}, v_{1}$, and $v_{2}$ are all colored differently, a permutation of colors allows the two colorings to combine and produce a 5-coloring of $G+\left[u_{1} v_{1}\right]+\left[u_{2} v_{2}\right]$.

In all other cases we can pick one vertex from each of the pairs $\left(u_{1}, v_{1}\right)$ and $\left(u_{2}, v_{2}\right)$ and have them not adjacent in $G$; by symmetry suppose the two are $u_{1}$ and $u_{2}$. Then since $G$ is a triangulation, $u_{1}$ and $u_{2}$ lie in different faces of $G-u_{1}-u_{2}$. Apply case $n=2$ of (3\&5CC) to $G-u_{1}-u_{2}$ and then notice that for each $u_{i}$ there is a color different than the colors of the 
neighbors of $u_{i}$ and (if it has been colored) $v_{i}$. Color first $u_{1}$ then $u_{2}$; this proves the theorem.

An obvious generalization of this proof gives

$\mathrm{V}$. Case $n$ of the (3\&5CC) implies that if $G$ is a planar graph with vertices $u_{i}, v_{i}(i=1,2, \cdots, n)$, and vertices $u_{i}$ are distinct and form an independent set, then $G^{*}$ formed from $G$ by adding all edges $\left[u_{i} v_{i}\right]$ can be 5-colored.

The simplest instance to which this theorem (supposing that (3\&5CC) is true) does not apply is the plane graph obtained by removing three edges from $K(6)$ of the form $\left[u_{i} v_{i}\right]$ for $i=1,2,3$. It will be seen that in no way can the labels be chosen to make $u_{1}, u_{2}, u_{3}$ a set of three independent vertices.

If it should turn out that ( $3 \& 5 C C)$ can be proved for a few more small values of $n$, but that with increasing $n$ the proof increases in difficulty, then this fact would tend to support the view that the (4CC) is false.

Afternote. The following related papers have come to our attention. The theorem proved in [4] can be shown equivalent to case $n=2$ of (3\&5CC) and hence implies the case $n=1$, which is our Theorem I. The proof in [4] is somewhat more difficult, which is in line with the prediction of the previous paragraph. In [5] can be found a result equivalent to Theorem I. Neither of these papers mention a connection between their results and [1]. In another paper [3], it is shown that the Connected Relative Coloring Conjecture is equivalent to the Four Color Conjecture.

\section{REFERENCES}

1. Paul C. Kainen, Relative colorings of graphs, J. Combinatorial Theory Ser. B 14 (1973), 259-262. MR 47 \#4840.

2. - A generalization of the 5-color theorem, Proc. Amer. Math. Soc. 45 (1974), 450-453.

3. Roy B. Levow, Relative colorings and the four-color conjecture, Amer. Math. Monthly 81 (1974), 491-492.

4. W. Meyer, Five-coloring planar maps, J. Combinatorial Theory Ser. B 13 (1972), 72-82. MR $46 \$ 7075$.

5. A. J. W. Hilton, R. Rado and S. H. Scott, $A(<5)$-colour theorem for planar graphs, Bull. London Math. Soc. 5 (1973), 302-306. CANADA

FACULTY OF MATHEMATICS, UNIVERSITY OF WATERLOO, WATERLOO, ONTARIO, 